\title{
Spinodal decomposition in a semidilute suspension of rodlike macromolecules
}

\author{
Jan Willem Winters and Theo Odijk* \\ Section Theory of Complex Fluids, Kluyver Laboratory of Biotechnology, Faculty of Applied Sciences, Delft University of Technology, \\ Julianalaan 67, 2628 BC Delft, The Netherlands \\ Paul van der Schoot ${ }^{\dagger}$ \\ Polymer Physics Group, Department of Applied Physics, Technische Universiteit Eindhoven, P.O. Box 513, \\ 5600 MB Eindhoven, The Netherlands \\ (Received 21 June 2000; published 18 December 2000)
}

\begin{abstract}
The Doi-Shimada-Okano [J. Chem. Phys. 88, 4070 (1988)] theory of the spinodal decomposition in a suspension of rods is reinvestigated analytically. Short enough rods may rotate fairly unhindered so we emphasize translational diffusion. The excluded-volume effect between the rods gives rise to a coupling of the orientational and translational degrees of freedom. This effect may be addressed correctly because the kernel in the equation describing the dynamic evolution of the one-particle distribution turns out to be degenerate. The two principal eigenvalues calculated analytically agree with previous numerical work by Maeda [Macromolecules 22, 1881 (1989); 23, 1464 (1990)]. It is concluded that the associated relaxation modes do not represent pure density and orientation fluctuation modes.
\end{abstract}

DOI: 10.1103/PhysRevE.63.011501

PACS number(s): 61.25.Hq, 05.20.Dd, 61.30.-v

A formally correct dynamical theory of a suspension of hard rods at the level of the second viral approximation was set up by Doi, Shimada, and Okano (DSO) a decade ago $[1,2]$. The major statistical problem is how to tackle the coupling of the orientational and translational degrees of freedom. The initial decay rate of the autocorrelation function of the scattered intensity of light is relatively easy to treat [1] in this respect. The predicted dependence of this rate on the scattering vector is in reasonable agreement with the rates, measured in scattering experiments on strongly entangled solutions of the semiflexible polymer $\operatorname{poly}(\gamma$-benzyl $\alpha$, L-glutamate) [3].

The issue of orientational-translational coupling was addressed for the static (equilibrium) version of the DSO theory [1], both numerically [4] and analytically, by variational methods [5]. The usefulness of variational principles is particularly evident when dealing with attractive interactions between the rods [6]. Maeda solved the dynamical DSO equations numerically in order to compute the dynamic scattering curves $[4,7]$. He pointed out that the two major modes had a peculiar dependence on the scattering vector, arising from the orientational-translational coupling inherent in the interaction kernel in the DSO theory [1]. Here we wish to introduce a way of tackling the eigenvalue problem by making use of the separability of the kernel in the limit where the rods are not strongly entangled. Our focus will be on the initial stage of the spinodal decomposition of the isotropic into the nematic liquid-crystalline phase.

Thirty to seventy nearby rods are needed to define a tube confining a test rod if the latter is to be regarded as entangled

\footnotetext{
*Author to whom correspondence should be addressed. Present address: P.O. Box 11036, 2301 EALeiden, The Netherlands. FAX: +31-71-5145346, Email address: odijktcf@wanadoo.nl

${ }^{\dagger}$ Also at: Dutch Polymer Institute, P.O. Box 902, 5600 AX Eindhoven, The Netherlands.
}

[8-11]. This means rodlike macromolecules with a relatively small aspect ratio $[L / b=O(10)$, where $L$ is the length and $b$ the diameter] rotate essentially without hindrance right up to and beyond the isotropic-nematic transition [12]. Recent experimental work on inorganic and anisometric colloids focuses on this type of nonentangled suspensions [13-15].

We consider an initially isotropic suspension of hard rods of number density $\nu$. We introduce the one-particle distribution $f(\vec{r}, \vec{u}, t)$ as a function of the position $\vec{r}$ and orientation $\vec{u}$ of a test rod at time $t$. For disentangled rods and with neglect of rotational diffusion, we may write a nonlinear diffusion equation for the distribution [1]

$$
\frac{\partial f}{\partial t}=D \frac{\partial}{\partial \vec{r}}\left[\frac{\partial f}{\partial \vec{r}}+f \frac{\partial M}{\partial \vec{r}}\right] .
$$

The translational diffusion constant $D$ here is set independent of the concentration, to a first approximation. The molecular field $M$ depends on the distribution $f$ and on the Mayer function $W$ for the excluded-volume interaction between two test rods [1]

$$
M\left(\vec{r}, \vec{r}^{\prime}, t\right)=\int d \vec{r}^{\prime} d \vec{u}^{\prime} W\left(\vec{r}-\vec{r}^{\prime}, \vec{u}, \vec{u}^{\prime}\right) f\left(\vec{r}^{\prime}, \vec{u}^{\prime}, t\right)
$$

The Mayer function $W\left(\vec{r}-\vec{r}^{\prime}, \vec{u}, \vec{u}^{\prime}\right)$ is unity if two rods with respective configurations $(\vec{r}, \vec{u})$ and $\left(\vec{r}^{\prime}, \vec{u}^{\prime}\right)$ overlap, and zero otherwise.

We now investigate the relaxation of small fluctuations in the distribution from equilibrium. In equilibrium we have $f$ $=\nu / 4 \pi$ if $f$ is normalized to the total number of rods in the suspension. If we write $f=(\nu / 4 \pi)+\delta f(\vec{r}, \vec{u}, t)$, where the fluctuation $\delta f$ is deemed small, and introduce its Fourier transform

$$
f_{\vec{k}}(\vec{u}, t)=\frac{1}{V} \int d \vec{r} \delta f(\vec{r}, \vec{u}, t) \exp [-i \vec{k} \cdot \vec{r}],
$$


we obtain a linear integrodifferential equation describing the time evolution of a fluctuation in the rod distribution:

$$
\frac{\partial f_{k}}{\partial t}=-\hat{\Omega}_{\vec{k}} f_{\vec{k}},
$$

The operator $\hat{\Omega}_{\vec{k}}$ is defined in terms of the Fourier transform $W_{\vec{k}}\left(\vec{u}, \vec{u}^{\prime}\right)$ of the Mayer function [1]

$$
\begin{gathered}
\hat{\Omega}_{\vec{k}} f_{\vec{k}}(\vec{u}, t)=D k^{2}\left[f_{\vec{k}}(\vec{u}, t)+\frac{\nu}{4 \pi} \int d \vec{u}^{\prime} W_{\vec{k}}\left(\vec{u}, \vec{u}^{\prime}\right) f_{\vec{k}}\left(\vec{u}^{\prime}, t\right)\right], \\
W_{\vec{k}}\left(\vec{u}, \vec{u}^{\prime}\right)=2 b L^{2} j_{0}(\vec{K} \cdot \vec{u})|\sin \gamma| j_{0}\left(\vec{K} \cdot \vec{u}^{\prime}\right),
\end{gathered}
$$

where $j_{0}(y) \equiv \sin y / y, \vec{K} \equiv \frac{1}{2} \vec{k} L$, and $\gamma=\gamma\left(\vec{u}, \vec{u}^{\prime}\right)$ is the angle between two test rods. In Eq. (6) we discern a term $2 b L^{2}|\sin \gamma|$, signifying the excluded volume between two test rods; translation-orientation coupling is expressed in the argument of the spherical Bessel functions $j_{0}$.

Solving Eq. (4) reduces to finding the spectrum of eigenvalues $\lambda$ of the equation

$$
\hat{\Omega}_{k} \Psi \equiv D k^{2}\left[\Psi+\frac{4 c}{\pi} \int_{-1}^{1} d x^{\prime} K\left(x, x^{\prime}\right) \Psi\left(x^{\prime}\right)\right]=\lambda \Psi,
$$

where a dimensionless concentration $c \equiv(\pi / 4) b L^{2} \nu$ has been introduced, and the kernel of the integral operator

$$
K\left(x, x^{\prime}\right)=\sum_{n=0}^{\infty} a_{2 n}(x) b_{2 n}\left(x^{\prime}\right)
$$

is written in terms of the functions $a_{2 n}(x) \equiv d_{2 n} b_{2 n}(x)$ and $b_{2 n}(x) \equiv j_{0}(k x) P_{2 n}(x)$. In Eqs. (7) and (8), $x \equiv \cos \theta$ and $x^{\prime} \equiv \cos \theta^{\prime}$ denote coordinates of two test rods, where the scattering vector $\vec{k}$ is chosen in the $z$ direction of our spherical coordinate system. We obtain Eq. (8) because one integration over the azimuthal angle yields a bilinear expansion in terms of Legendre polynomials, $P_{2 n}$,

$$
\frac{1}{2 \pi} \int_{0}^{2 \pi} d \varphi|\sin \gamma|=\sum_{n=0}^{\infty} d_{2 n} P_{2 n}\left(x^{\prime}\right) P_{2 n}(x),
$$

with expansion coefficients $d_{0}=\pi / 4, d_{2}=-5 \pi / 32, \ldots$ [5]. We next note that the kernel in the integral equation (7) is separable [16]. Hence we posit a solution of the form

$$
\Psi(x)=\sum_{m=0}^{\infty} \varphi_{2 m} a_{2 m}(x),
$$

with coefficients $\varphi_{2 m}$ to be determined. Upon inserting Eq. (10) into Eq. (7), we note that the functions $a_{2 m}(x)$ are linearly independent [except possibly at the zeros of $j_{0}(k x)$, their total measure equalling zero]. Thus the eigenvalue statement is equivalent to

$$
\sum_{n=0}^{\infty} I_{2 m, 2 n} \varphi_{2 n}=\Lambda \varphi_{2 m},
$$

$$
I_{2 m, 2 n} \equiv \int_{-1}^{1} d x a_{2 m}(x) b_{2 n}(x), \quad \Lambda \equiv \frac{\pi}{4 c}\left(\frac{\lambda}{D k^{2}}-1\right) .
$$

The eigenvalues of the resulting matrix equation may be found by solving

$$
\operatorname{det}(\vec{I}-\Lambda \vec{e})=0,
$$

where $\vec{e}$ denotes the unit matrix, and $\vec{I}$ a matrix with elements $I_{2 m, 2 n}$ defined in Eq. (11).

In order to study the onset of spinodal decomposition, we need to know if some eigenvalue $\lambda$ is negative for certain $k$. We focus on a two-mode approximation involving the two major modes ( $m=0$ and 1$)$, as Maeda already showed numerically that the eigenvalues beyond $m=1$ are essentially purely diffusive [4]. The relevant eigenfunctions are

$$
\Psi_{ \pm}=\varphi_{0}^{ \pm} a_{0}(x)+\varphi_{2}^{ \pm} a_{2}(x) .
$$

Equation (12) then gives for the eigenvalues $\Lambda_{+}$and $\Lambda_{-}$ associated with the hybrid eigenfunctions $\Psi_{+}$and $\Psi_{-}$,

$$
\Lambda_{ \pm}=\frac{1}{2}\left(I_{00}+I_{22}\right) \pm \frac{1}{2} \sqrt{\left(I_{00}-I_{22}\right)^{2}+4 I_{02} I_{20}},
$$

with

$$
\begin{gathered}
I_{00}=2 d_{0}\left[K^{-1} \operatorname{Si}(2 K)-j_{0}^{2}(K)\right], \\
I_{02}=d_{0}\left[2 K^{-2}-\frac{1}{2} K^{-2} \cos 2 K-\frac{3}{4} K^{-3} \sin 2 K\right. \\
\left.-K^{-1} \operatorname{Si}(2 K)\right], \\
I_{20}=d_{2} I_{02} / d_{0}, \\
I_{22}=\frac{d_{2}}{16 K^{5}}\left[-16 K^{3}+2 K\left(2 K^{2}-9\right) \cos 2 K+9 \sin 2 K\right. \\
\left.-6 K^{2} \sin 2 K+8 K^{4} \operatorname{Si}(2 K)\right],
\end{gathered}
$$

and $\operatorname{Si}(x)$ the usual sine integral $\operatorname{Si}(x)=\int_{0}^{x}(\sin t / t) d t$. It is easy to show that $\Lambda_{+}$is positive and $\Lambda_{-}$is negative for all scattering vectors, by using the fact that $\left[P_{2}(x)-P_{2}(y)\right]^{2}$ $\geqslant 0$ for all $x$ and $y$ within the interval $-1 \leqslant x, y \leqslant 1$. The functions $\Lambda_{+}$and $\Lambda_{-}$are shown in Figs. 1 and 2. Note the oscillatory behavior of $\Lambda_{-}$at high $K$, which is perhaps unexpected for a theory within the second virial approximation. The original eigenvalues expressing measurable relaxation rates are given by

$$
\begin{aligned}
& \lambda_{+}=D k^{2}\left(1+\frac{4 c}{\pi} \Lambda_{+}\right), \\
& \lambda_{-}=D k^{2}\left(1+\frac{4 c}{\pi} \Lambda_{-}\right) .
\end{aligned}
$$

These rates are pictured in Figs. 3 and 4, and agree well with the values determined numerically by Maeda [4]. (See the entries for modes 0 and 2 in his Table III; there is a slight disparity because Maeda also accounted for parallel and perpendicular diffusion, as he discussed himself [4].) 




FIG. 1. Dimensionless eigenvalue $\Lambda_{+}$as a function of the dimensionless magnitude of the scattering vector $K$.

It is at small scattering angles only that the eigenfunctions given by Eq. (13) collapse into "pure" density and orientation modes. Indeed, only for $k L \rightarrow 0$ do we find that $\Psi_{+}$ $\rightarrow P_{0}(x)$ and $\Psi_{-} \rightarrow P_{2}(x)$, the former representing a pure density fluctuation mode and the latter a pure orientation fluctuation mode. (Here uninteresting numerical prefactors have been ignored.) At wave vectors greater than zero, the density and orientational fluctuations intermingle, to give rise to the hybrid + and - modes. The hybrid + mode is always stable: $\lambda_{+}>0$ for all $k$ and $c$. At low scattering angles

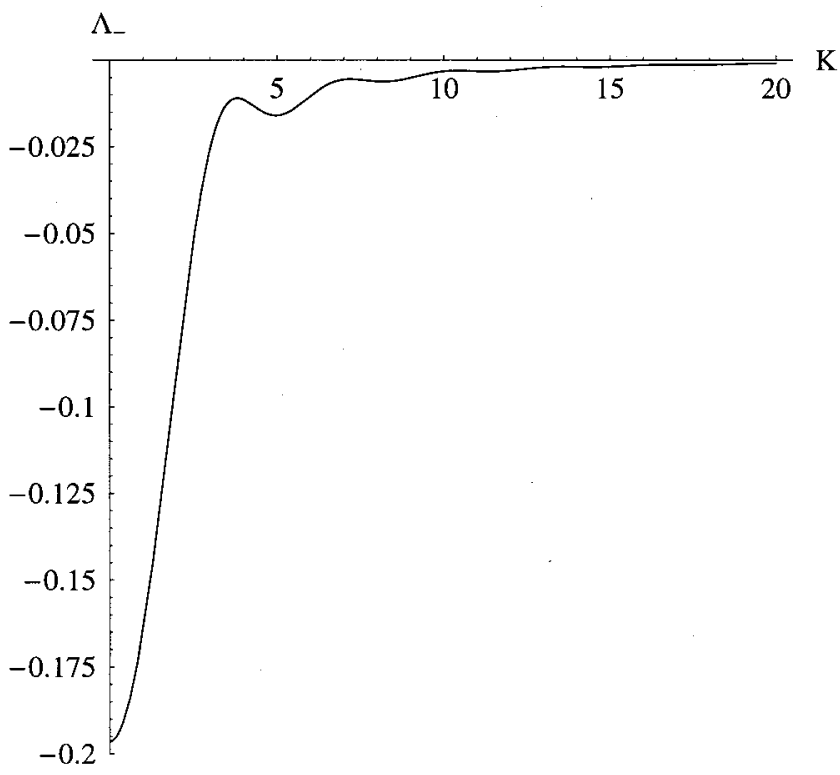

FIG. 2. Dimensionless eigenvalue $\Lambda_{-}$as a function of the dimensionless magnitude of the scattering vector $K$.

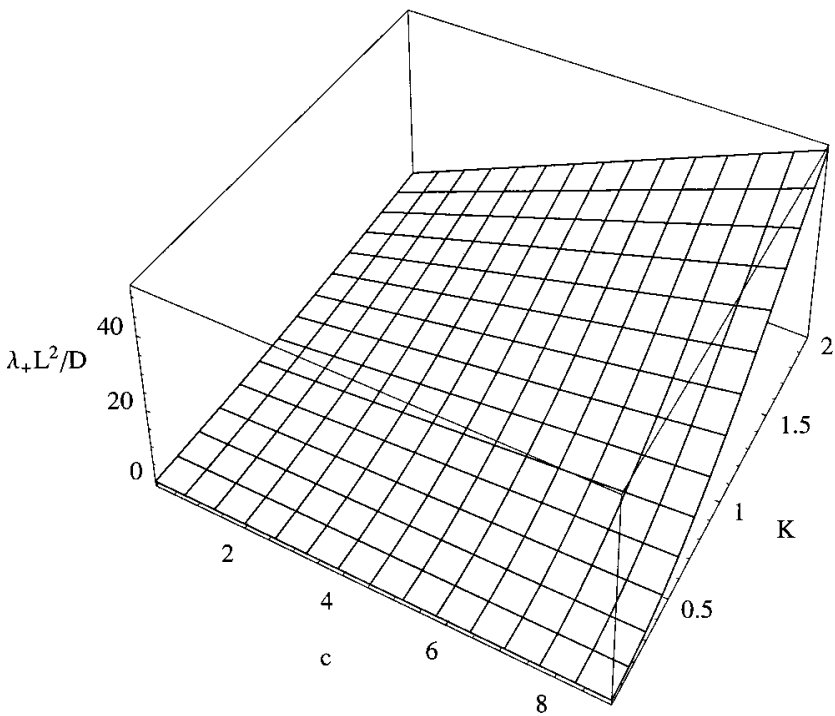

FIG. 3. Dimensionless relaxation rate $\lambda_{+}$as a function of the dimensionless concentration $c$, and the dimensionless magnitude of the scattering vector $K$.

$k L \ll 1$, the rate $\lambda_{+}$reduces to that of a simple cooperative diffusion process

$$
\lambda_{+}=D k^{2}\left[1+2 c+O\left(k^{2} L^{2}\right)\right] .
$$

The - mode can become unstable at sufficiently high densities. To order $k^{4}$, the eigenvalue $\lambda_{-}$has a form analogous to that in Cahn's theory of the spinodal decomposition in simple liquids [17] [Note that a surface tension is implicit in Eq. (2)]:

$$
\lambda_{-}=D k^{2}\left(1-\frac{C}{4}+\frac{11}{252} c K^{2}+\cdots\right)
$$

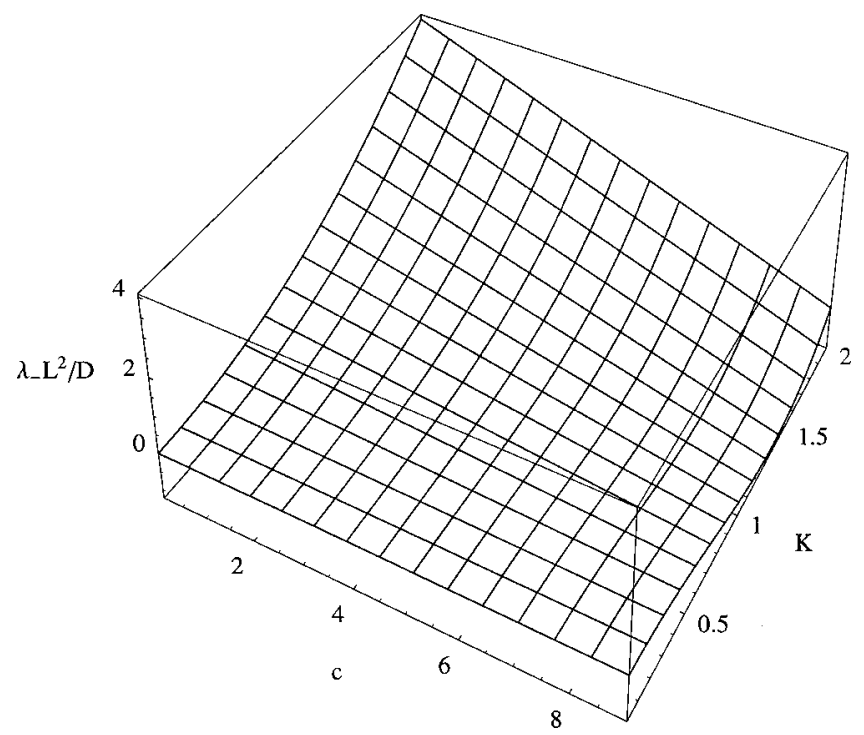

FIG. 4. Dimensionless relaxation rate $\lambda_{-}$as a function of the dimensionless concentration $c$, and the dimensionless magnitude of the scattering vector $K$. This mode becomes unstable for $c>4$. 
This has a minimum at

$$
K_{m}=\frac{1}{2} k_{m} L=\left[\left(\frac{1}{4}-\frac{1}{c}\right) \frac{126}{11}\right]^{1 / 2}
$$

Accordingly, we infer that fluctuations of the hybrid form $\Psi_{-}$, centered around $k_{m}$ and of approximate size $2 \pi / k_{m}$, grow out of an initially isotropic state at concentrations $c$ $>4$, that is, exceeding the spinodal concentration [18].
In conclusion, we have been able to establish the full $k$ dependence of the initial stage of the spinodal decomposition of a suspension of rods. We have used a systematic twomode approximation, different from that used previously, to our knowledge, and we have assumed that the rods are short enough to rotate freely. We hope to include dispersion forces into the analysis above, so as to discuss recent experiments on coated boehmite particles [15]. We have to be wary of the practical import of attractive forces even when they are small [6].
[1] M. Doi, T. Shimada, and K. Okano, J. Chem. Phys. 88, 4070 (1988).

[2] T. Shimada, M. Doi, and K. Okano, J. Chem. Phys. 88, 7181 (1988).

[3] L. M. DeLong and P. S. Russo, Macromolecules 24, 6139 (1991). See the review by P. S. Russo, in Dynamic Light Scattering, edited by W. Brown (Oxford University Press, Oxford, 1993).

[4] T. Maeda, Macromolecules 22, 1881 (1989).

[5] P. van der Schoot and T. Odijk, Macromolecules 23, 4181 (1990).

[6] P. van der Schoot and T. Odijk, J. Chem. Phys. 97, 515 (1992). See the review by T. Odijk, in Light Scattering, Principles and Development, edited by W. Brown (Oxford University Press, Oxford, 1996).

[7] T. Maeda, Macromolecules 23, 1464 (1990).

[8] M. Fixman, Phys. Rev. Lett. 54, 337 (1985).
[9] G. T. Keep and R. Pecora, Macromolecules 18, 1167 (1985).

[10] I. Teraoka, Y. Mori, N. Ookubo, and R. Hayakawa, Phys. Rev. Lett. 55, 2712 (1985).

[11] J. J. Magda, H. T. Davis, and M. Tirrell, J. Chem. Phys. 85, 6674 (1986).

[12] T. Odijk, Macromolecules 21, 3511 (1988).

[13] M. P. B. van Bruggen, H. N. W. Lekkerkerker, and J. K. G. Dhont, Phys. Rev. E 56, 4394 (1997).

[14] M. P. B. van Bruggen, H. N. W. Lekkerkerker, G. Maret, and J. K. G. Dhont, Phys. Rev. E 58, 7668 (1998).

[15] M. P. B. van Bruggen, J. K. G. Dhont, and H. N. W. Lekkerkerker, Macromolecules 32, 2256 (1999).

[16] R. P. Kanwal, Linear Integral Equations, 2nd ed. (Birkhäuser, Boston, 1997).

[17] J. W. Cahn, J. Chem. Phys. 42, 93 (1965).

[18] R. F. Kayser and H. J. Raveché, Phys. Rev. A 17, 2067 (1978). 